\title{
Breaking the Crowther Limit with "Sudoku" Tomography: Combining Depth- Sectioning and Tilt Series for High-Resolution, Wide-Field Reconstructions
}

Robert Hovden ${ }^{1}$, Peter Ercius ${ }^{2}$, Yi Jiang ${ }^{3}$, Deli Wang ${ }^{4}$, Yingchao Yu ${ }^{4}$, Héctor D. Abruña ${ }^{4}$, Veit Elser $^{3}$, David A. Muller ${ }^{1,}$

1) School of Applied and Engineering Physics \& Kavli Institute at Cornell University, Ithaca, NY

2) National Center for Electron Microscopy, Lawrence Berkeley National Laboratory, Berkeley, CA

3) Department of Physics, Cornell University, Ithaca, NY

4) Department of Chemistry and Chemical Biology, Cornell University, Ithaca, NY

With electron beams smaller than the bond length of hydrogen, aberration-corrected scanning transmission electron microscopes (STEM) can currently image materials with resolutions below the shortest bond length in nature. However, these atomic resolution images are only $2 \mathrm{D}$ projections of a specimen. In order to determine the full 3D structure, one must acquire a series of images over a range of specimen tilts. Missing information from the physical restrictions on the specimen tilt range and finite tilt increments diminishes the resolution of a 3D STEM tomogram [1]. For most objects (> $20 \mathrm{~nm}$ ), this typically limits volumetric resolutions of electron tomography to roughly $1 \mathrm{~nm}$ - twenty times worse than the best resolution in 2D projections [2]. Surprisingly, aberration-corrected electron microscopes underperform compared to uncorrected instruments: high-resolution $(<1 \mathrm{~nm})$ tomography of general extended objects, to date, has not been possible with aberration-corrected instruments because the depth-of-focus $(5-10 \mathrm{~nm})$ of aberration-corrected STEMs is smaller than most objects of interest today. The sub-Angstrom resolution of aberration-corrected electron microscopes is accompanied by a strongly diminished depth of focus, causing regions of "large" specimens $(>5 \mathrm{~nm})$ to appear blurred or missing [3]. High-resolution, 3D reconstructions of extended objects that take advantage of the improved resolution of aberration-correctors requires collecting information beyond a traditional tilt series.

Here we present a generalized-"Sudoku" tomography scheme that adds depth (through-focal) sectioning (the Sudoku sub-volume constraints) to traditional tilt-tomography (the Sudoku projection constraints) to reconstruct extended objects, with high-resolution in all three dimensions. For traditional (S)TEM tomography the images at every tilt-angle must correspond to a perfect projection of the original object and thus the entire specimen must always be in focus. Each projection corresponds to a plane of information in reciprocal space (Fig 1b). The finite number of tilts causes small wedges of missing information between each tilt that limit the resolution and $3 \mathrm{D}$ volume of a reconstructed object. However, through-focal tomography utilizes additional information provided by a through-focal series (Fig. 1a) at every tilt (Fig 1c), not only overcoming the limitations of a small depth-of-focus, but also filling in the smaller missing wedges of information present between each tilt and improving the quality of the $3 \mathrm{D}$ reconstruction (Fig. $2 \mathrm{~b}$ vs $2 \mathrm{~d}$ ). This effectively decouples the limiting Crowther relationship between 3D resolution, object size, and number of tilts.

Using the $30 \mathrm{mrad}$ convergence angle of the $300 \mathrm{keV}$ aberration-corrected TEAM I instrument, porous $\mathrm{PtCu}$ nanoparticles lying on an extended 3D carbon support were imaged [4]. A through-focal series acquired at every tilt overcame the microscope's diminished depth-of-focus and provided a continuum of high-frequency detail in the tomogram (Fig. 2a,b). Interconnected pore structures resulting from chemical treatment was observed in most $\mathrm{PtCu}$ nanoparticles throughout the extended support $(\sim 390 \mathrm{~nm})$. Traditional tomographic methods were not able to reconstruct the particles with sufficient detail to observe pores in all particles (Fig. 2c,d), demonstrating the utility of through-focal approaches to aberration-corrected STEM tomography. 
[1] Midgley, P. A., Weyland, M. et al, Chem. Commun. 907-908 (2001).

[2] Crowther, R. A., DeRosier, D. J. \& Klug, A. Proc. R. Soc. A 317, 319-340 (1970).

[3] Hovden, R., Xin, H. L. \& Muller, D. A. Microsc. Microanal. 17, 75-80 (2011).

[4] Wang, D. et al. Nano Lett. 12, 5230-5238 (2012).

[5] Tomography funded by CCMR, an NSF MRSEC (DMR-1120296) and LBNL supported by DOE (\#DE-AC02-05CH11231)..Catalyst samples funded by EMC2, a DOE BES EFRC (DE-SC0001086).
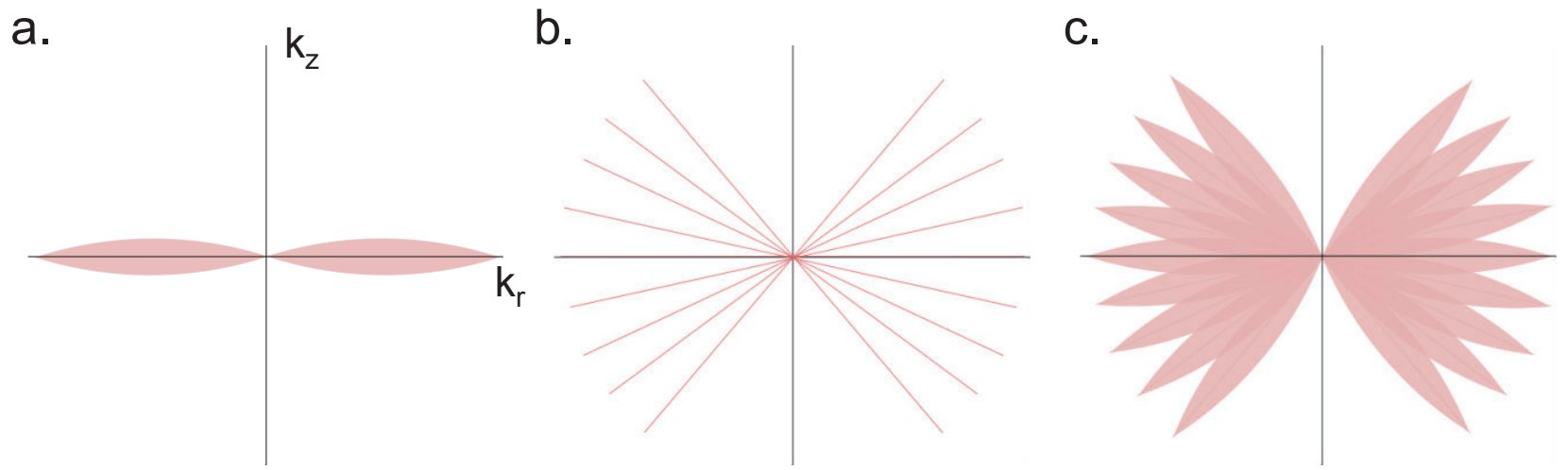

Figure 1: Three diagrams show how various 3D reconstructions fill in information in reciprocal space: a) a slice of the rotationally symmetric contrast transfer function (CTF) of an electron probe represents the information obtained from a through focal series. b) Slice through planar discs of information gained by traditional electron tomography. A finite number of tilts and a limited tilt range fail to sample much available information. c) Through-focal tomography combines approaches a) \& b) to significantly increase the information sampled at each tilt.

Through-Focal Tomography
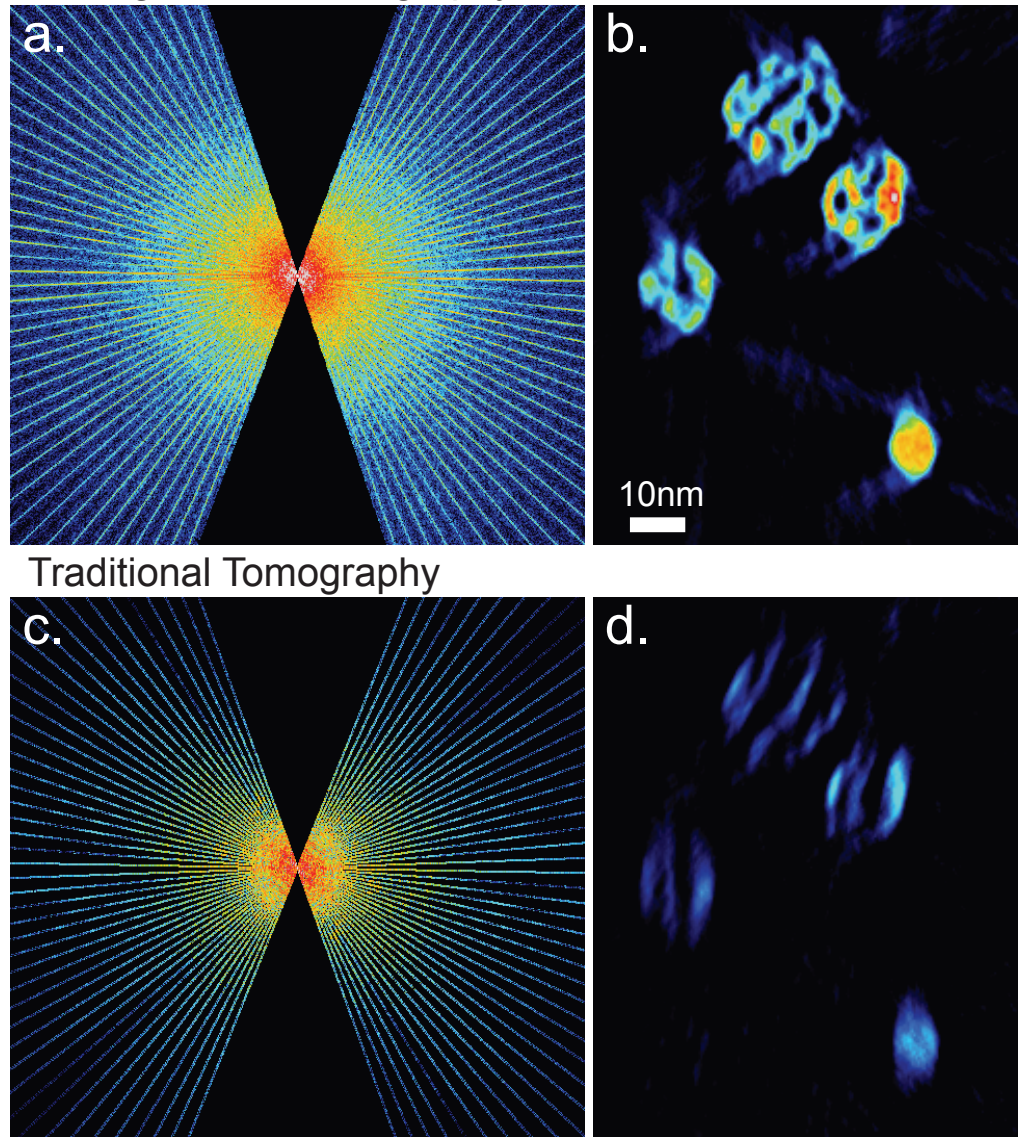

Figure 2: Experimental comparison of an unweighted through-focal tomography and a traditional unweighted tomography of porous $\mathrm{PtCu}$ nanoparticles. In Fourier space (a) through-focal tomography acquires a continuum of information throughout the microscope's tilt range. In the realspace reconstruction, dramatic differences in the two techniques can be seen in the nanoparticle structure (b vs. d). A cross section of several nanoparticles shows clear presence of pores in the through-focal reconstruction (b); features that are missing or too heavily distorted in the traditional reconstruction $(\mathrm{d})$. 